\title{
Clinicopathological Characteristics of Breast Cancer in a Population of Eastern Algeria
}

\author{
Type of article: Conference abstract \\ Aicha Boulkrah1, Sarra Touaibia1, Hajira Berredjem1 , Amel Boulmaiz 1, Rachid Cheniki 2,3, Jihen \\ Rima Benaziza 2,3, Nardjess Chadli 2,3 , Nadia Kermiche 2,3 , Hayet Aouras 2,3 \\ 1 Université Badji Mokhtar-Annaba, Faculté des Sciences, Département de Biochimie, \\ Laboratoire de Biochimie et Microbiologie Appliquées, Annaba, Algeria \\ 2 Université Badji Mokhtar-Annaba, Faculté de Médecine, Annaba, Algeria \\ 3 Hôpital public EHS Abdallah Nouaouria EL Bouni, Annaba, Algeria \\ Corresponding Author: hajira.berrejem@univ-annaba.dz.
}

\begin{abstract}
Background: One of the most common cancers in female is the breast cancer. It is the most important cause of neoplasie deaths among women in both developed countries and developing countries. The incidence of breast cancer has increased more than 10000 new cases per year. The aim of our study is to determine the clinical characteristics of breast cancer in the Eastern Algeria.

Methods: This is a retrospective study made in public hospital EHS Abdallah Nouaouria El Bouni, Annaba gynecology and anatomy pathology services, which included 40 patients over a period of three months from July to September 2019.

Results: The mean age was $49.87(\mathrm{SD}=9.2)$ years. The age group of $40-76$ years represented the majority of the study population (85\%). A total of 30 patients $(75 \%)$ had invasive ductal carcinoma, while $3(7,5 \%)$ had ductal carcinoma in situ, $3(7,5 \%)$ had inflammatory breast cancer, $2(5 \%)$ had mucinous carcinoma and $2(5 \%)$ had phyllodes tumors .

The average size tumor was $3,7 \mathrm{~cm}$. The rate of tumor grade with large size T2, T3 and T4b was $70 \%$. $45 \%$ of patients had positive lymph nodes and $2,5 \%$ had bilateral breast cancer. No metastatic cases were reported.

Results also show that the most affected women are between 35 and 55 years; $92,5 \%$ have an invasive breast cancer, and more than $80 \%$ are in advanced stage .

Conclusion: Histological diagnosis is important since it state the specific type of carcinoma. One of the best approaches to prevent this disease is early diagnosis.

Keywords: Breast cancer, age, clinicopathological characteristics.
\end{abstract}

\section{Conflict of interest statement}

This article is a conference abstract presented at the International Congress on Health Sciences and Medical Technologies, Tlemcen Algeria 5-7 December 2019, ICHSMT' 19.

\section{Authors' biography}

No Biography.

\section{References}

No references 